\title{
Inter-Collicular Suppression Compresses All Types of Rate-Amplitude Functions of Inferior Collicular Neurons in Mice
}

\author{
Liang CHENG ${ }^{1,2}$, Hui-Xian MEI ${ }^{1}$, Yun HUANG ${ }^{2}$ \\ ${ }^{1}$ School of Life Sciences and Hubei Key Laboratory of Genetic Regulation and Integrative Biology, \\ Central China Normal University, Wuhan, China, ${ }^{2}$ School of Psychology and Key Laboratory of \\ Adolescent Cyberpsychology and Behavior of Ministry of Education, Central China Normal \\ University, Wuhan, China
}

Received September 5, 2015

Accepted January 22, 2016

On-line April 12, 2016

\section{Summary}

The two inferior colliculi (IC) are paired structures in the midbrain that are connected to each other by a bundle of commissural fibers. The fibers play an important role in coordinating sound signal processing between the two inferior colliculi. This study examined inter-collicular suppression on sound signal processing in amplitude domain of mice by measuring the rate-amplitude functions (RAFs) of neurons in one IC during the electrical stimulation of the opposite IC. Three types (monotonic, saturated and non-monotonic) RAFs of collicular neurons were measured before and during inter-collicular suppression. Inter-collicular suppression significantly increased the slope, decreased the dynamic range and narrowed down the responsive amplitude of all RAFs to high amplitude level but did not change the type of most (36/43, $84 \%)$ RAFs. As a result, all types of RAFs were compressed at a greater degree at low than at high sound amplitude during inter-collicular suppression. These data indicate that inter-collicular suppression improve sound processing in the high amplitude domain.

\section{Key words}

Inter-collicular suppression - Rate-amplitude function • Amplitude-coding • Inferior colliculus

\section{Corresponding author}

L. Cheng, School of Psychology, Central China Normal University, Luoyu Street 152, Wuhan 430079, China. E-mail: ChengL@mail.ccnu.edu.cn

\section{Introduction}

The inferior colliculi (IC) are paired mammalian structures in the midbrain that receive excitatory and inhibitory ascending and descending projections and are also connected to each other by a bundle of fibers called the commissure of IC (CoIC) (Aitkin and Phillips 1984, Syka and Popeláŕ 1984, Herrera et al. 1987, Oliver et al. 1991, Saldana and Merchan 1992, Malmierca et al. 1995, 2009, Moore et al. 1997, Popeláŕ et al. 2003, Cant and Benson 2006, Hernández et al. 2006, Winer 2006). CoIC fibers include point-to-point connections between the corresponding frequency laminae of the two ICs as well as divergent connections projecting from one IC neuron to a wide range of frequency laminae in the opposite IC (Malmierca et al. 1995, 2009). These connections provide the final opportunity for functional interactions between the two sides of the auditory pathway at the subcortical level.

In vitro studies have demonstrated that microelectrical stimulation (ES) of CoIC fibers elicits both excitatory and inhibitory postsynaptic potentials (EPSPs and IPSPs, respectively) in IC neurons (Smith 1992, Moore et al. 1998). Similarly, blocking CoIC fibers in vivo by injecting kynurenic acid (a nonspecific glutamatergic antagonist) into one IC changes the number of impulses and the frequency-response area of neurons located in the corresponding frequency laminae of the opposite IC (Malmierca et al. 2003, 2005, Orton and Rees 2014). Such inter-collicular interactions through 
CoIC provide opportunity for modulation during ascending auditory processing in multiple parametric domains including frequency and amplitude (Mei et al. 2012a,b, 2013, Cheng et al. 2013). In amplitude domain, inter-collicular interactions modulate the response magnitude and the rate-amplitude function (RAF) of collicular neurons and change the minimal threshold (MT) and dynamic range (DR) through the interplay between focused facilitation and widespread suppression in the CoIC (Mei et al. 2012a). Widespread intercollicular suppression increases the sensitivity of IC neurons to minor changes over a narrower range of sound amplitude while focused facilitation produces the opposite effect (Mei et al. 2012b).

To further study the inter-collicular interaction on sound processing in amplitude domain, we examine the effect of electrical stimulation of one IC on the RAF of the neurons in the other IC. Specifically, we examine if the degree of inter-collicular suppression during electrical stimulation of one IC may vary with the type of affected collicular neurons in the other IC.

\section{Methods}

All experiments were approved by the Institutional Animal Care and Use Committee of Central China Normal University and complied with the Guide for the Care and Use of Laboratory Animals (NIH Publication No. 85-23, revised 1996).

\section{Animal preparation and surgery}

As described in our previous studies (Mei et al. 2012a, Cheng et al. 2013), a flat head of a $1.8 \mathrm{~cm}$ nail was glued onto the exposed skull of each of 21 Nembutal-anesthetized (60-90 mg/kg b. wt.) Kunming mice (Mus musculus, 20-25 g, b. wt.) with acrylic glue and dental cement. After securing the mouse to an aluminum plate with a plastic band inside a soundproof room (at a temperature of $28-30{ }^{\circ} \mathrm{C}$ ), its head was immobilized by a set of screw. Small holes (diameter: 200-500 $\mu \mathrm{m}$ ) were made in the skull above each IC. A $2 \mathrm{M} \mathrm{NaCl}$ glass pipette electrode (tip diameter: $<1 \mu \mathrm{m}$, impedance: 5-10 M $\Omega$ ) was orthogonally inserted into one IC to record sound activated responses while a pair of custom-made bipolar tungsten electrodes (see below) was inserted into the other IC for focal electrical stimulation (ES) and recording sound activated responses of stimulated IC neuron.
Stimulation and isolation of acoustically evoked collicular (IC) neurons

For acoustic stimulation (AS), continuous sine sound waves from a function generator (GFG-8016G, Good Will Inst Co., Ltd, Bayan Lepas, Penang, Malaysia) were formed into $40 \mathrm{~ms}$ pure tone ( $5 \mathrm{~ms}$ rise-decay times) with custom-made tone burst generator (electronic switch) driven by a stimulator (Model SEN-7203, Nihon Kohden Co, Shinjuku, Tokyo, Japan). The tone pulses were then amplified (custom-made amplifier) after passing a decade attenuator (LAT-45, Leader, Kohokuku, Yokohama, Japan) before they were fed into a small loudspeaker (AKG model $\mathrm{CK} 50,1.5 \mathrm{~cm}$ in diameter, $1.2 \mathrm{~g}$, frequency response $1-100 \mathrm{kHz}$ ). The loudspeaker was placed $30 \mathrm{~cm}$ away from the mouse ear and $60^{\circ}$ contralateral to the recording site. Calibration of the loudspeaker was conducted with a $1 / 4$ inch microphone (4939, B\&K, Denmark) placed at the mouse's ear using a measuring amplifier (2610, B\&K, Denmark). The output of the loudspeaker was expressed in decibel sound pressure level (dB SPL) in reference to $20 \mu \mathrm{Pa}$ root mean square. The maximal available sound amplitude ranged from $95 \mathrm{~dB}$ to $110 \mathrm{~dB}$ SPL between 10 and $80 \mathrm{kHz}$ but dropped off sharply to $80 \mathrm{~dB}$ SPL at $100 \mathrm{kHz}$ thereafter.

Two insulated tungsten electrodes (FHC Inc, Bodowin, ME, USA) were glued together (glue 502, inter-tip distance: $\leq 100 \mu \mathrm{m}$ ) to form a pair of custommade tungsten electrodes. These electrodes were used for recording sound activated IC responses and for focal electrical stimulation in the IC stimulating site (4 ms train of four monophasic pluses of $0.1 \mathrm{~ms}$ with $0.9 \mathrm{~ms}$ plusegap at 2 trains/s, 5-50 $\mu \mathrm{A}$ ) using stimulator (Model SEN7203, Nihon Kohden Co, Shinjuku, Tokyo, Japan) and stimulus isolation unit (CSS-202J, Nihon Kohden Co, Tokyo, Japan).

Upon isolation of an IC neuron in stimulating side (abbreviated as $\mathrm{IC}_{\mathrm{ES}}$ neuron) using a pair of custommade tungsten electrodes with $40 \mathrm{~ms}$ pure tone at 2 pulses/s, its best frequency (BF) and MT were audiovisually determined by changing the frequency and sound amplitude. The sound frequency that elicited the neuron's response at the lowest amplitude was defined as the BF. The threshold at the BF was defined as the MT. At the $\mathrm{MT}$, the neuron, on average, responded with $50 \%$ probability to $\mathrm{BF}$ pulses. Acoustically evoked responses of an IC neuron in the recording side (abbreviated as $\mathrm{IC}_{\mathrm{Rec}}$ neuron) was then isolated with a $2 \mathrm{M} \mathrm{NaCl}$ glass electrodes. After determining the BF and MT of this $\mathrm{IC}_{\mathrm{Rec}}$ neuron, its response to $\mathrm{BF}$ sound pulses delivered at 
$10 \mathrm{~dB}$ above the MT was recorded as a control response. The neuron's response was then monitored again during $\mathrm{ES}$ of the $\mathrm{IC}_{\mathrm{ES}}$ neuron isolated before. The ES was delivered between 5 and $50 \mu \mathrm{A}$ and at a randomly chosen inter-pulse interval (IPI, interval between AS and ES). The current level was gradually increased in order to find an $\mathrm{IC}_{\mathrm{Rec}}$ neuron affected by the $\mathrm{IC}_{\mathrm{ES}} \mathrm{ES}$ and to observe the effect on response of the $\mathrm{IC}_{\mathrm{Rec}}$ neuron under different current level. Then, the ES current was fixed at moderate level $(25 \mu \mathrm{A}$, high enough and without too much diffusion, Jen and Zhou 2003) and the IPI was adjusted systematically to determine the optimal IPI during which the ES would produce maximal effect. If the percent decrease in number of impulses of $\mathrm{IC}_{\mathrm{Rec}}$ neuron induced by focal ES did not reach $30 \%$, the $\mathrm{IC}_{\mathrm{Rec}}$ neuron was abandoned. Otherwise it was regarded as a modulated $\mathrm{IC}_{\mathrm{Rec}}$ neuron by inter-collicular suppression. At the optimal IPI, the RAF of $\mathrm{IC}_{\mathrm{Rec}}$ neuron was then measured before and during $\mathrm{ES}$ in $\mathrm{IC}_{\mathrm{ES}}$. A RAF was measured with the neuron's number of impulses obtained at MT and $10 \mathrm{~dB}$ increments above the MT with $40 \mathrm{~ms}$ BF sound. The best amplitude (BA) was defined as the specific amplitude which elicited the maximum in the neuron's number of impulses for a specific frequency. The dynamic range (DR) of RAF was defined as the amplitude range from $10 \%$ below the maximum to $10 \%$ above the minimum in the neuron's number of impulses. The middle DR (mDR) was defined as the middle amplitude value of DR. The slope of a RAF was obtained by dividing the percent change in the neuron's number of impulses within the dynamic range by the dynamic range and expressed in \%/dB.

\section{Data collection and analysis}

Recorded action potentials were amplified and sent to a computer for acquisition of post-stimulus-time histograms (PSTH) (bin width: $250 \mu \mathrm{s}$; sampling period: $150 \mathrm{~ms}$ ) to 32 stimuli. The total number of impulses in each histogram was used to quantify the neuron's response under each stimulation condition.

The suppressive effect on the RAFs of an affected $\mathrm{IC}_{\mathrm{Rec}}$ neuron during the focal electrical stimulation of the opposite IC (i.e. $\mathrm{IC}_{\mathrm{ES}}$ ) was determined by calculating the percent decrease in the control number of impulses of the $\mathrm{IC}_{\mathrm{Rec}}$ neuron. All data processed and plotted using Sigma Plot 2000. They were then quantitatively examined and statistically compared using SPSS 13.0 (one-way and repeated measures ANOVA at $P<0.05$, Student's $t$-test and paired $t$-test at $P<0.05$ ).

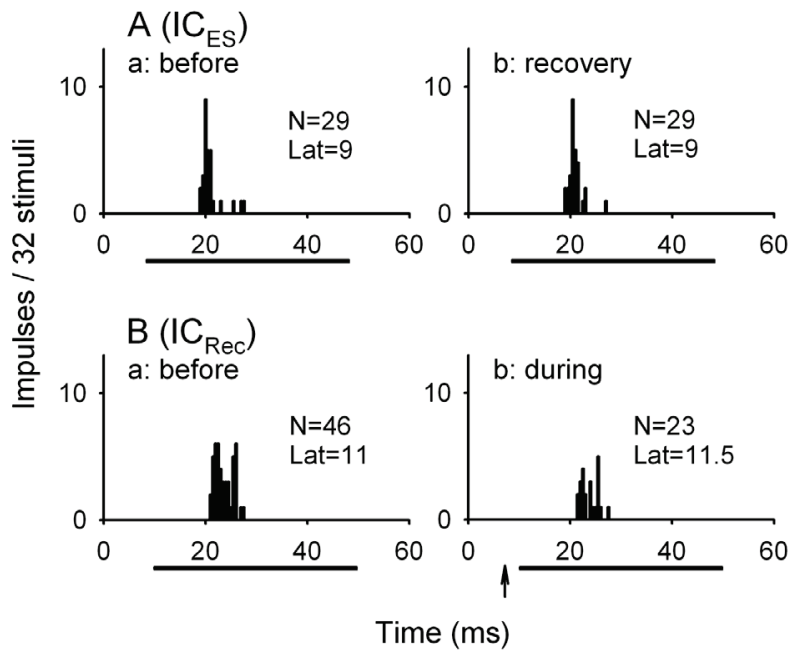

Fig. 1. The response of two representative $I C_{E S}$ and $I C_{\text {Rec }}$ neurons under different stimulation condition. (A) The response of a representative $\mathrm{IC}_{\mathrm{ES}}$ neuron obtained before (a) and recovery (b) from focal electrical stimulation (ES). (B) The response of a representative $\mathrm{IC}_{\mathrm{Rec}}$ neuron obtained before (a) and during (b) focal ES. The response of all these two neurons were obtained with a best frequency (BF) sound delivered at $10 \mathrm{~dB}$ above the minimal threshold (MT). N, number of spikes; Lat, latency; Horizontal bar: acoustic stimulus. Arrow: focal electrical stimulation. The $\mathrm{BF}, \mathrm{MT}$ and recording depth of this neuron were $17.1 \mathrm{kHz}$ ， $58 \mathrm{~dB}$ SPL， $1670 \mu \mathrm{m}(\mathrm{A}) ; 15.6 \mathrm{kHz}, 68 \mathrm{~dB} \mathrm{SPL}$, $1510 \mu \mathrm{m}$ (B); respectively.

\section{Results}

The responses of $43 \mathrm{IC}_{\mathrm{Rec}}$ neurons were recorded during sound stimulation and their responses were suppressed during focal electrical stimulation of the opposite $\mathrm{IC}_{\mathrm{ES}}$ (Fig. 1Ba vs. b). Recording depth ranged from 227 to $2003 \mu \mathrm{m}$ (mean \pm SD: $1083 \pm 401 \mu \mathrm{m}$ ), the BFs from 5.5 to $27.6 \mathrm{kHz}(14.2 \pm 4.8 \mathrm{kHz})$, and the MTs from 15 to $87 \mathrm{~dB}$ SPL ( $54 \pm 17 \mathrm{~dB}$ SPL). Focal ES did not appear to affect the normal acoustically evoked response properties of $\mathrm{IC}_{\mathrm{ES}}$ neurons, which recovered to the control level after ES ceased (Fig. 1Aa vs. b). The RAFs of 43 neurons can be described as three groups, monotonic, saturated and non-monotonic. In the monotonic group $(n=19,44.2 \%)$, the neuron's number of impulses monotonically increased with sound amplitude (Fig. 2A-2). In the saturated group ( $\mathrm{n}=12,27.9 \%)$, the neuron's number of impulses increased with sound amplitude up to a maximum point, but then leveled out and did not increase more than $25 \%$ at higher sound amplitudes (Fig. 2B-2). In the non-monotonic group $(n=12,27.9 \%)$, the neuron's number of impulses increased with sound amplitude up to a maximum point and then decreased more than $25 \%$ at higher amplitudes (Fig. 2C-2). 

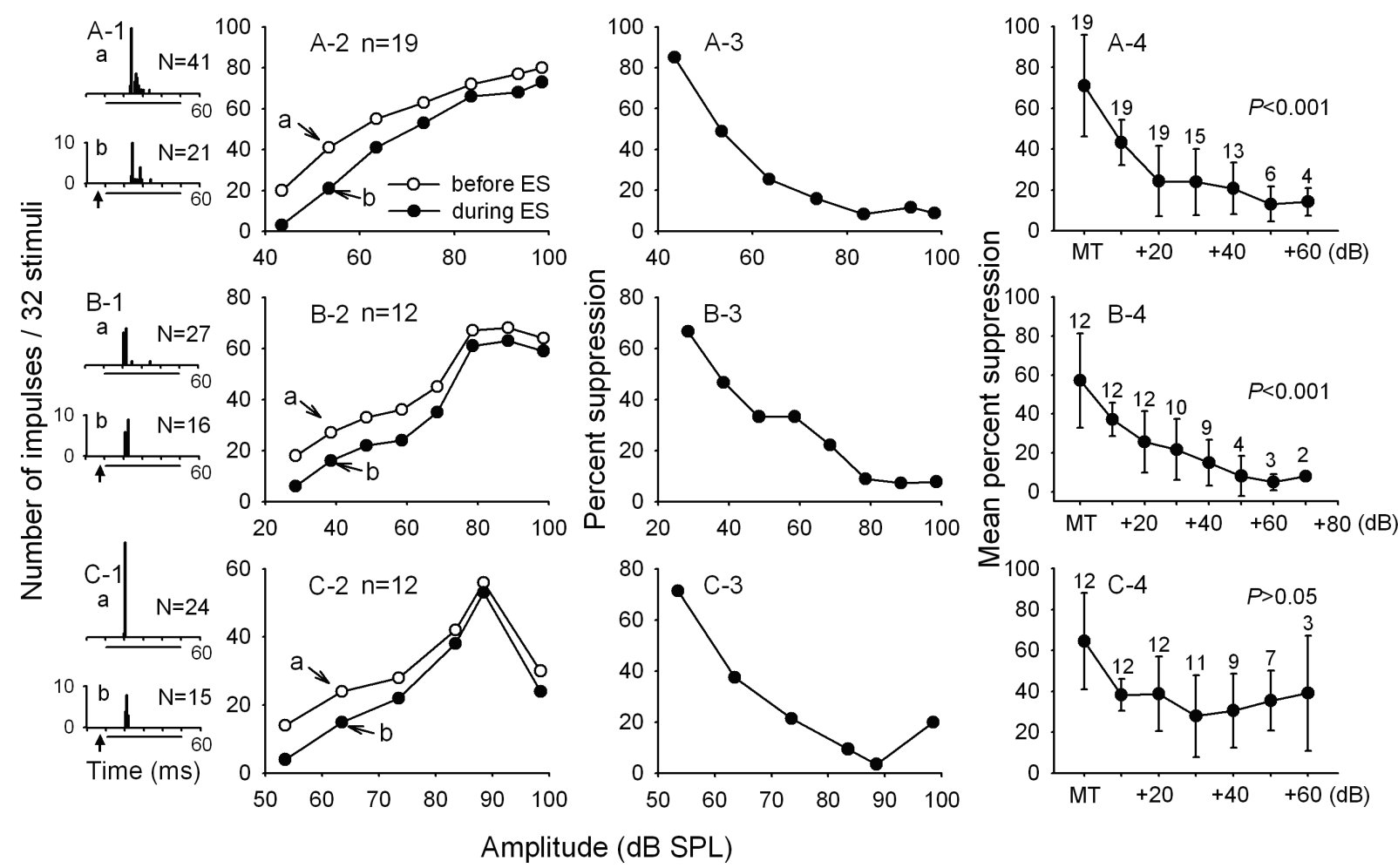

Fig. 2. Suppressive modulation of rate-amplitude functions of three types of recorded $I C$ (IC $\left.C_{R e c}\right)$ neurons $(\mathbf{A}, \mathbf{B}, \mathbf{C})$ during focal $I C_{E S} E S$. $(\mathbf{A - 1}, \mathbf{B}-1, \mathbf{C}-1)$ Post-stimulus-time histograms of responses from three representative $\mathrm{IC}_{\mathrm{Rec}}$ neurons to best frequency (BF) sounds (horizontal bar under abscissa) delivered at $10 \mathrm{~dB}$ above each neuron's minimal threshold (MT) before (A-1a, B-1a, C-1a; arrows on A-2a, B-2a, C-2a) and during (up-arrow under abscissa; A-1b, B-1b, C-1b; arrows on A-2b, B-2b, C-2b) focal IC ES ES. N, number of impulses. (A-2, B-2, C-2) Rate-amplitude functions (RAFs) (monotonic, saturated, and non-monotonic) of the three representative $\mathrm{IC}_{\mathrm{Rec}}$ neurons before (unfilled circle) and during (filled circle) focal $\mathrm{IC}_{\mathrm{ES}} \mathrm{ES}$. $\mathrm{n}$, number of neurons. (A-3, B-3, $\mathbf{C}-\mathbf{3}$ ) Percent suppression of the number of impulses caused by focal IC $C_{E S} E S$ for the three representative $I_{\text {Rec }}$ neurons at different stimulus amplitudes. (A-4, B-4, C-4) Mean percent suppression of the number of impulses caused by focal IC $C_{E S}$ ES for the three types of IC $C_{\text {Rec }}$ RAFs at different stimulus amplitudes. Numbers above each standard deviation bar indicate number of neurons. The $P$-value was obtained after a one-way ANOVA. The BFs, MTs, and recording depths of these three neurons were $16.7 \mathrm{kHz}, 44 \mathrm{~dB} \mathrm{SPL}, 1270 \mu \mathrm{m}(\mathrm{A}) ; 13.6 \mathrm{kHz}$, $29 \mathrm{~dB}$ SPL, $1691 \mu \mathrm{m}(\mathrm{B}) ; 13.5 \mathrm{kHz}, 54 \mathrm{~dB}$ SPL, $1011 \mu \mathrm{m}(\mathrm{C})$.

Figure 2A-1, B-1, and C-1 shows the PSTHs of three representative $\mathrm{IC}_{\mathrm{Rec}}$ neurons obtained with $\mathrm{BF}$ sound delivered at $10 \mathrm{~dB}$ above each neuron's MT before and during ES. Figure $2 \mathrm{~A}-2$, B-2, and $\mathrm{C}-2$ shows the $\mathrm{RAFs}$ of these three neurons before and during $\mathrm{IC}_{\mathrm{ES}} \mathrm{ES}$. It is clear that the percent inter-collicular suppression in the number of impulses of affected $\mathrm{IC}_{\mathrm{Rec}}$ neurons typically decrease with stimulus amplitude progressively increased above the MT. At the very high stimulus amplitude, percent suppression in the number of impulses reached a plateau level for $\mathrm{IC}_{\mathrm{Rec}}$ neurons with the monotonic and saturated RAFs (Fig. 2A-3, B-3, A-4, B-4). However, the percent suppression in the number of impulses further increased at still high sound amplitude for $\mathrm{IC}_{\mathrm{Rec}}$ neurons with non-monotonic RAFs (Fig. 2C-3, C-4). We further studied the effect of inter-collicular suppression on these non-monotonic neurons by dividing the mean percent suppression in Figure 2C-4 into two parts based on the stimulus amplitude at which the mean percent suppression reversed its decreasing trend (Malmierca et al. 2005): part one, with percent suppression obtained $\leq 20 \mathrm{~dB}$ above MT; part two, with percent suppression obtained $\geq 30 \mathrm{~dB}$ above MT (Fig. 3). Statistical analysis showed that the mean percent suppression in the part one was greater than that in the part two $(P<0.001$, Student's $t$-test $)$, suggesting intercollicular suppression in the number of impulses of affected non-monotonic $\mathrm{IC}_{\mathrm{Rec}}$ neurons was stronger at low than at high sound amplitude, similar to monotonic and saturated $\mathrm{IC}_{\mathrm{Rec}}$ neurons.

To study the inter-collicular suppression on sound processing in amplitude domain, we examine if intercollicular suppression during electrical stimulation of one IC may change the type of RAF of affected neurons in the other IC. Table 1 compares the type of RAF of these $\mathrm{IC}_{\mathrm{Rec}}$ neurons before and during $\mathrm{ES}$ of the opposite $\mathrm{IC}_{\mathrm{ES}}$. It is clear that the RAF of most $\mathrm{IC}_{\mathrm{Rec}}$ neurons remained unchanged during $\mathrm{ES}$ of the opposite $\mathrm{IC}_{\mathrm{ES}}$. 


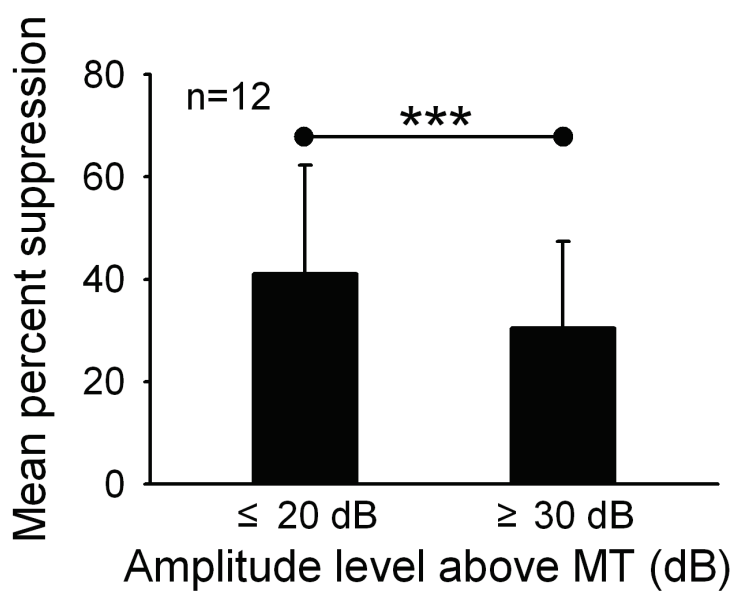

Fig. 3. Mean percent suppression of spikes in non-monotonic $I_{\text {Rec }}$ neurons during focal $\mathrm{IC}_{\mathrm{ES}} \mathrm{ES}$. Bars show the mean percent suppression in spikes caused by focal $\mathrm{IC}_{\mathrm{ES}} \mathrm{ES}$ for two ranges of stimulus amplitude: $\leq 20 \mathrm{~dB}$ above each neuron's MT and $\geq 30 \mathrm{~dB}$ above each neuron's MT. n, the number of neurons; $* * *, P<0.001$ (paired $t$-test).
We further studied the effect of inter-collicular suppression on the RAF of affected $\mathrm{IC}_{\mathrm{Rec}}$ neurons in one IC by comparing the MT, BA, DR, $\mathrm{mDR}$ and slope of their RAF before and during electrical stimulation of the opposite $\mathrm{IC}_{\mathrm{ES}}$. Regardless of the type of the RAF of affected $\mathrm{IC}_{\mathrm{Rec}}$ neurons, focal electrical stimulation of the $\mathrm{IC}_{\mathrm{ES}}$ elevated the MT (Fig. 4A-1, B-1, C-1, $P<0.001$, Student's paired $t$-test), decreased the DR (Fig. 4A-3, B-3, C-3, $P<0.001$, Student's paired $t$-test), shifted the mDR toward a high-stimulus amplitude (Fig. 4A-4, B-4, C-4, $P<0.01-0.001$, Student's paired $t$-test), and increased the slope (Fig. 4A-5, B-5, C-5, $P<0.05-0.01$, Student's paired $t$-test) of the RAF of the $\mathrm{IC}_{\operatorname{Rec}}$ neurons. There was no significant different in the degree of inter-collicular suppression effect on these parameters of the RAF of affected $\mathrm{IC}_{\mathrm{Rec}}$ neurons according to their type of $\mathrm{RAF}$ (Table 2, $P>0.05$, one-way ANOVA).

Table 1. Types of RAFs of $I C_{\text {Rec }}$ neurons before and during focal IC $C_{E S} E S$.

\begin{tabular}{lcccc}
\hline & Before ES & \multicolumn{3}{c}{ During ES } \\
\cline { 2 - 5 } & $\mathrm{n}$ & Monotonic & Saturated & Non-monotonic \\
& & $\mathrm{n}$ & $\mathrm{n}$ & $\mathrm{n}$ \\
\hline Monotonic & $19(44.2)$ & $15(34.9)$ & $3(7.0)$ & $1(2.3)$ \\
Saturated & $12(27.9)$ & $2(4.6)$ & $10(23.3)$ & $0(0)$ \\
Non-monotonic & $12(27.9)$ & $17(39.5)$ & $1(2.3)$ & $11(25.6)$ \\
Total & $43(100)$ & $14(32.6)$ & $12(27.9)$ \\
\hline
\end{tabular}

$\mathrm{n}$, number of $\mathrm{IC}_{\mathrm{Rec}}$ neurons. Number in parentheses indicates the percentage of $\mathrm{I} \mathrm{C}_{\mathrm{Rec}}$ neurons.

Table 2. Comparison of percent change in MT, BA, DR, mDR and Slope across three types of RAFs of $I_{\text {Rec }}$ neurons due to IC stimulation.

\begin{tabular}{|c|c|c|c|c|c|}
\hline $\mathbf{n}$ & & $\begin{array}{c}\text { Monotonic } \\
19\end{array}$ & $\begin{array}{c}\text { Saturated } \\
12\end{array}$ & $\begin{array}{c}\text { Non-monotonic } \\
12\end{array}$ & $P$ \\
\hline \multirow{2}{*}{$M T(d B S P L)$} & Range & $1.3-38.9$ & $1.3-58.8$ & $0-48$ & \\
\hline & Mean \pm SD & $11.4 \pm 8.9$ & $15.2 \pm 16.7$ & $13.6 \pm 13.1$ & $>0.05$ \\
\hline \multirow{2}{*}{$B A(d B S P L)$} & Range & $0-10.5$ & $0-10.5$ & $0-35.7$ & \\
\hline & Mean \pm SD & $0.9 \pm 2.7$ & $1.8 \pm 3.4$ & $4.0 \pm 10.6$ & $>0.05$ \\
\hline \multirow{2}{*}{$D R(d B)$} & Range & $10.1-59.6$ & $4.5-68.9$ & $1.9-73.5$ & \\
\hline & Mean \pm SD & $28.3 \pm 15.6$ & $25.6 \pm 21.9$ & $37.0 \pm 22.8$ & $>0.05$ \\
\hline \multirow{2}{*}{$m D R(d B)$} & Range & $0.5-14.0$ & $1.7-17.4$ & $2.0-48.3$ & \\
\hline & Mean \pm SD & $5.7 \pm 4.1$ & $7.9 \pm 6.0$ & $12.7 \pm 12.7$ & $>0.05$ \\
\hline \multirow{2}{*}{ Slope $(\% / d B)$} & Range & $5.2-81.4$ & $0.2-253.3$ & $5.8-153.9$ & \\
\hline & Mean \pm SD & $33.3 \pm 22.5$ & $50.6 \pm 72.0$ & $44.1 \pm 43.2$ & $>0.05$ \\
\hline
\end{tabular}

n, number of $\mathrm{IC}_{\mathrm{Rec}}$ neurons; $P$, significant level (one-way ANOVA). 
$\circ \sqsubset$ before ES

- during ES
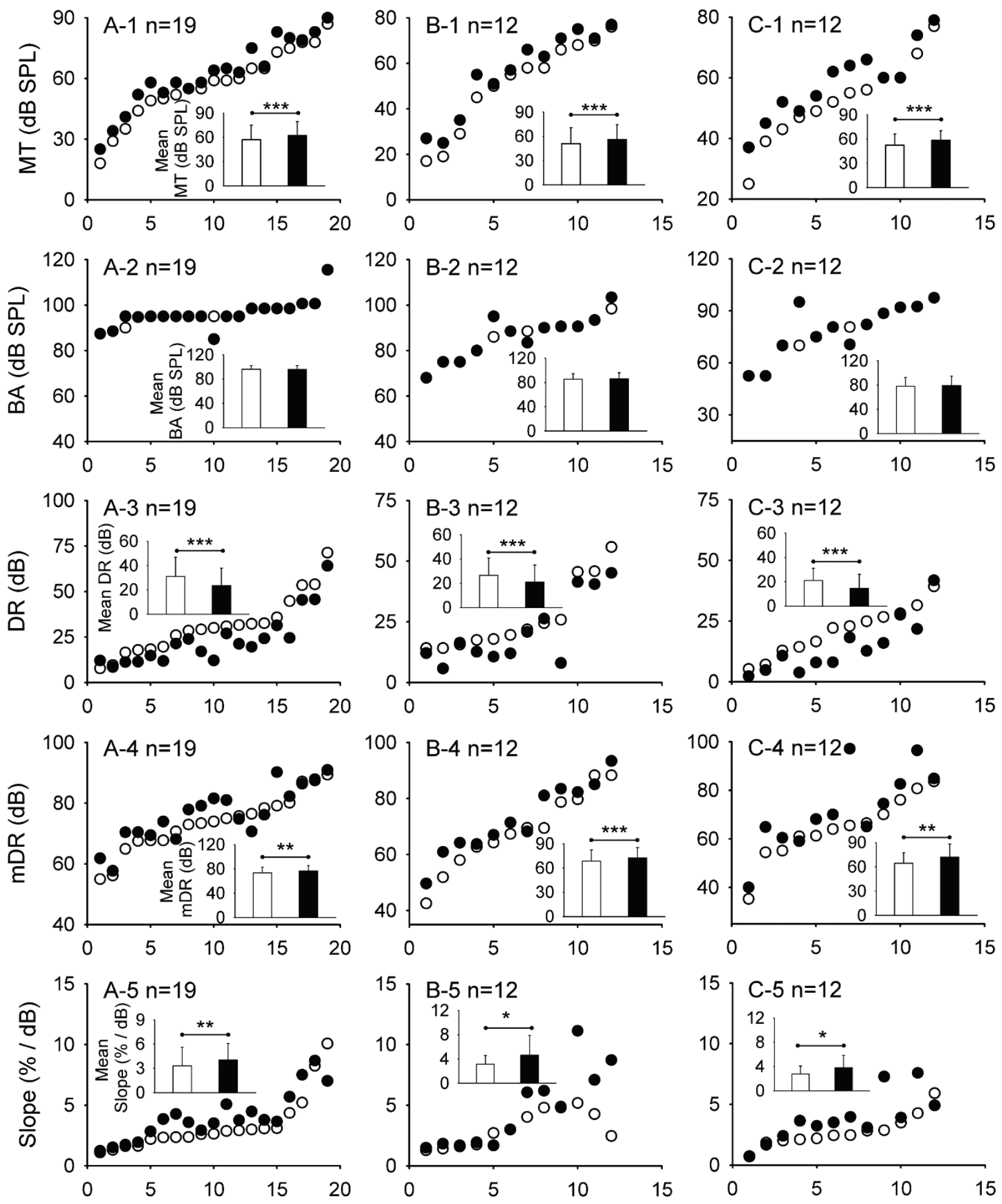

Neuron number

Fig. 4. Distribution of different parameters for the three types of $I C_{R e c}$ neurons before and after focal $I C_{E S} E S$. $A-1-A-5, B-1-B-5$, C-1 - C-5 show the distribution of MT, BA, DR, mDR, and slope of RAFs for the three types of $I_{\text {Rec }}$ neurons ( $A$, monotonic; $\mathrm{B}$, saturated; $\mathrm{C}$, non-monotonic) before (unfilled circles) and during (filled circles) focal $\mathrm{IC}_{\mathrm{ES}} \mathrm{ES}$. The bars in each panel are the mean value of each parameter. $\mathrm{n}$, the number of neurons. $*, P<0.05, * *, P<0.01, * * *, P<0.001$ (paired $t$-test). 


\section{Discussion}

In the present study, we examined the effect of inter-collicular suppression on signal processing in amplitude domain using focal electrical stimulation in one IC and electrophysiological recording in the other IC. We used a focal electrical stimulus of $25 \mu \mathrm{A}$ that has been proved effective and appropriate for studying intercollicular modulation and corticofugal modulation of collicular signal processing (Jen et al. 1998, 2003, Mei et al. 2012a,b, Cheng et al. 2013). As such, the acoustically evoked responses of electrically stimulated neuron recovered quiet well after cessation of electrical stimulation (Fig. 1A). Under such $\mathrm{IC}_{\mathrm{ES}}$ electrical stimulation, inter-collicular suppression was activated and the number of impulses of $\mathrm{IC}_{\mathrm{Rec}}$ neurons were suppressed (Fig. 1B).

The inter-collicular suppression compresses the RAFs of the collicular neurons over a range of soundstimulus amplitudes (Fig. 2A-2, B-2, C-2) and the degree of compression was greater at low than at high sound stimulus amplitude (Fig. 2A-3, B-3, A-4, B-4, Fig. 3). Conceivably, this observation is probably due to the fact that inter-collicular suppression produces a constant amount of inhibitory input to $\mathrm{IC}_{\mathrm{Rec}}$ neurons at all sound stimulus amplitude but the effectiveness of suppression progressively decreases when the excitatory input to $\mathrm{IC}_{\mathrm{Rec}}$ neurons increases with sound amplitude. These indicate that inter-collicular suppression involve in modulating sound-amplitude processing in IC neurons by suppressing the neuron's number of impulses at low-sound-stimulus amplitudes. The similar observations have been reported in previous studies that show the inter-collicular interaction can modulate facilitory and inhibitory effects on collicular neurons and the greatest effects occurs at near-threshold amplitude levels (Malmierca et al. 2005, Mei et al. 2012a).

Consistent with previous studies, here we observed that IC neurons had three types of RAFs: monotonic, saturated, and non-monotonic (Fig. 2A-2, B-2, C-2) (Phillips and Kelly 1989, Zhou and Jen 2002, $\mathrm{Wu}$ and Jen 2009). Inter-collicular suppression did not induce changes in the type of most RAFs of the $\mathrm{IC}_{\mathrm{Rec}}$ neurons (Table 1). According to previous studies, we know that RAFs (i.e. amplitude tuning) are created primarily by imbalanced synaptic inhibition that is disproportionately large at high-sound-stimulus amplitudes (Oswald et al. 2006, Wu et al. 2006, Tan et al. 2007, 2009, Zhou et al. 2012). The inter-collicular suppression here results in less suppression at high sound-stimulus amplitudes, which would not usually change the RAF type of these IC neurons (Plontke et al. 1999, Wu and Jen 2007, 2009). However, we did observe a few instances in which $\mathrm{IC}_{\mathrm{ES}} \mathrm{ES}$ did result in a change in RAF type (Table 1). This could have resulted from inhibitory local circuits that become more active with greater acoustic stimulation.

What is the biological significance of intercollicular suppression in sound-signal processing in each type of IC neuron? The increase in MT, decrease in the DRs, but stable in the BA cause the slope of RAFs increased and the responsive amplitudes narrowed down to high amplitude level. Such alterations would sharpen the sensitivity of all types of IC neurons to variation in high sound amplitude within a narrower range (Fig. 4). Conceivably, the inter-collicular suppression could improve the sensitivity of IC neurons to high amplitude sound as well as to variation in amplitude such as amplitude modulated sound (Rees and Møller 1987, Joris et al. 2004, Dean et al. 2005). As such, inter-collicular suppression might come into play when the IC neurons receive and encode the high-amplitude acoustic information. However, the alterations on RAFs did not differ cross RAF types, suggesting that these effects of inter-collicular suppression on auditory sensitivity do not depend on the RAF types. The inter-collicular suppression appears to function similarly with inhibitory corticofugal control that has been shown to improve sound-amplitude signal processing of subcortical auditory structures such as the IC, medial geniculate body (MGB), and cochlear nucleus (CN) (Jen et al. 1998, Suga et al. 2000, Zhou and Jen 2000, 2002, He 2003, Ma and Suga 2007, Luo et al. 2008). Presumably, in the IC, the intercollicular suppression might work with corticofugal inhibition together to modulate the auditory sensitivity of neurons at the same time. In addition, inter-collicular suppression might also help maintain the unilateral dominance of one IC by suppressing the acoustic-evoked responses of neurons in the opposite IC, thus shaping sensitivity to interaural intensity differences, which are needed for sound localization at the azimuth and for binaurally stereoscopic hearing (Irvine et al. 1996, Konishi 2000, Grothe 2003, Malmierca et al. 2005, Grothe et al. 2010). Future studies will be needed to test these predictions.

In this study, the effects of inter-collicular suppression on sound amplitude processing were examined using focal electrical stimulation in one IC and 
electrophysiological recording in the other IC. The CoIC fibers as the direct pathway from one IC to the other would be activated directly and primarily when electrically stimulating the unilateral IC, which can efficiently mediate the inter-collicular suppression observed in this study (Aitkin and Phillips 1984, Oliver et al. 1991, Malmierca et al. 2009, Cheng et al. 2013). However, there is another possible pathway that can mediate the inter-collicular interactions that is activation of indirect neural circuit involving other auditory nuclei (e.g. corticofugal feedback loop). It is necessary in the future study to test the possible neural pathway by inactivation of ipsilateral auditory cortex with Lidocaine, or ablation of the CoIC during electrical stimulation of IC.

In conclusion, inter-collicular suppression significantly increased the slope, decreased the dynamic range and narrowed down the responsive amplitude of all RAFs to high amplitude level but did not change the type of RAFs. As a result, all types of RAFs were compressed at a greater degree at low than at high sound amplitude during inter-collicular suppression. These data indicate that inter-collicular suppression can improve sound processing of IC neurons in the high amplitude domain regardless of their RAF type.

\section{Conflict of Interest}

There is no conflict of interest.

\section{Acknowledgements}

We thank the anonymous reviewers for commenting on an earlier version of this manuscript. We are particularly grateful to Prof. Chen Q.C. for his scientific and technical support. This work is supported by grants from National Science Foundation of China (\#30970972, \#31300914) and China Postdoctoral Science Foundation (\#2013M531719, \#2014T70716) and the Fundamental Research Funds for Central Universities (CCNU14A05046) and a grant from Key Laboratory of Adolescent Cyberpsychology and Behavior of Ministry of Education, Central China Normal University (2014B10).

\section{References}

AITKIN LM, PHILLIPS SC: The interconnections of the inferior colliculi through their commissure. J Comp Neurol 228: $210-216,1984$.

CANT NB, BENSON CG: Organization of the inferior colliculus of the gerbil (Meriones unguiculatus): differences in distribution of projections from the cochlear nuclei and the superior olivary complex. J Comp Neurol 495: 511-528, 2006.

CHENG L, MEI HX, TANG J, FU ZY, JEN PHS, CHEN QC: Bilateral collicular interaction: modulation of auditory signal processing in frequency domain. Neuroscience 235: 27-39, 2013.

DEAN I, HARPER NS, MCALPINE D: Neural population coding of sound level adapts to stimulus statistics. Nat Neurosci 8: 1684-1689, 2005.

GROTHE B: New roles for synaptic inhibition in sound localization. Nat Rev Neurosci 4: 540-550, 2003.

GROTHE B, PECKA M, MCALPINE D: Mechanisms of sound localization in mammals. Physiol Rev 90: 983-1012, 2010.

HE J: Corticofugal modulation of the auditory thalamus. Exp Brain Res 153: 579-590, 2003.

HERNÁNDEZ O, REES A, MALMIERCA MS: A GABAergic component in the commissure of the inferior colliculus in rat. Neuroreport 17: 1611-1614, 2006.

HERRERA M, SANCHEZ DEL CAMPO F, SMITH-AGREDA V: The commissural nucleus of the inferior colliculus in the rabbit. A morphological study. J Hirnforsch 28: 671-683, 1987.

IRVINE DR, RAJAN R, AITKIN LM: Sensitivity to interaural intensity differences of neurons in primary auditory cortex of the cat. I. types of sensitivity and effects of variations in sound pressure level. J Neurophysiol 75 : 75-96, 1996.

JEN PH, ZHOU X: Corticofugal modulation of amplitude domain processing in the midbrain of the big brown bat, Eptesicus fuscus. Hear Res 184: 91-106, 2003.

JEN PH, CHEN QC, SUN XD: Corticofugal regulation of auditory sensitivity in the bat inferior colliculus. J Comp Physiol A 183: 683-697, 1998. 
JORIS PX, SCHREINER CE, REES A: Neural processing of amplitude-modulated sounds. Physiol Rev 84: 541-577, 2004.

KONISHI M: Study of sound localization by owls and its relevance to humans. Comp Biochem Physiol A 126: 459-469, 2000.

LUO F, WANG QZ, KASHANI A, YAN J: Corticofugal modulation of initial sound processing in the brain. $J$ Neurosci 28: 11615-11621, 2008.

MALMIERCA MS, REES A, LE BEAU FE, BJAALIE JG: Laminar organization of frequency-defined local axons within and between the inferior colliculi of the guinea pig. J Comp Neurol 357: 124-144, 1995.

MALMIERCA MS, HERNANDEZ O, FALCONI A, LOPEZ-POVEDA EA, MERCHÁN M, REES A: The commissure of the inferior colliculus shapes frequency response areas in rat: an in vivo study using reversible blockade with microinjection of kynurenic acid. Exp Brain Res 153: 522-529, 2003.

MALMIERCA MS, HERNANDEZ O, REES A: Intercollicular commissural projections modulate neuronal responses in the inferior colliculus. Eur J Neurosci 21: 2701-2710, 2005.

MALMIERCA MS, HERNANDEZ O, ANTUNES FM, REES A: Divergent and point-to-point connections in the commissural pathway between the inferior colliculi. J Comp Neurol 514: 226-239, 2009.

MA XF, SUGA N: Multiparametric corticofugal modulation of collicular duration-tuned neurons: modulation in the amplitude domain. $J$ Neurophysiol 97: 3722-3730, 2007.

MEI HX, CHENG L, TANG J, FU ZY, WANG X, JEN PHS, CHEN QC: Bilateral collicular interaction: Modulation of auditory signal processing in amplitude domain. PLoS One 7: e41311, 2012a.

MEI HX, CHENG L, TANG J, FU ZY, JEN PHS, CHEN QC: Modulation of amplitude sensitivity by bilateral collicular interaction among different frequency laminae. Neurosci Lett 517: 13-17, $2012 \mathrm{~b}$.

MEI HX, CHENG L, CHEN QC: Neural interactions in unilateral colliculus and between bilateral colliculi modulate auditory signal processing. Front Neural Circuit 7: 68, 2013.

MOORE DR, KOTAK VC, SANES DH: Commissural and lemniscal synaptic input to the gerbil inferior colliculus. J Neurophysiol 80: 2229-2236, 1998.

MOORE JK, GUAN YL, SHI SR: Axogenesis in the human fetal auditory system, demonstrated by neurofilament immunohistochemistry. Anat Embryol (Berl) 195: 15-30, 1997.

OLIVER DL, KUWADA S, YIN TC, HABERLY LB, HENKEL CK: Dendritic and axonal morphology of HRP-injected neurons in the inferior colliculus of the cat. J Comp Neurol 303: 75-100, 1991.

ORTON LD, REES A: Intercollicular commissural connections refine the representation of sound frequency and level in the auditory midbrain. eLife 3: $03764,2014$.

OSWALD AM, SCHIFF ML, REYES AD: Synaptic mechanisms underlying auditory processing. Curr Opin Neurobiol 16: 371-376, 2006.

PHILLIPS DB, KELLY JB: Coding of tone-pulse amplitude by single neurons in auditory cortex of albino rats (Rattus norvegicus). Hear Res 37: 269-279, 1989.

PLONTKE SK, LIFSHITZ J, SAUNDERS JC: Distribution of rate-intensity function types in chick cochlear nerve after exposure to intense sound. Brain Res 842: 262-274, 1999.

POPELÁŘ J, NWABUEZE-OGBO FC, SYKA J: Changes in neuronal activity of the inferior colliculus in rat after temporal inactivation of the auditory cortex. Physiol Res 52: 615-628, 2003.

REES A, MØLLER AR: Stimulus properties influencing the responses of inferior colliculus neurons to amplitudemodulated sounds. Hear Res 27: 129-143, 1987.

SALDANA E, MERCHAN MA: Intrinsic and commissural connections of the rat inferior colliculus. $J$ Comp Neurol 319: 417-437, 1992.

SMITH PH: Anatomy and physiology of multipolar cells in the rat inferior collicular cortex using the in vitro brain slice technique. J Neurosci 12: 3700-3715, 1992.

SUGA N, GAO E, ZHANG Y, MA X, OLSEN JF: The corticofugal system for hearing: recent progress. Proc Natl Acad Sci USA 97: 11807-11814, 2000.

SYKA J, POPELÁ $\breve{R}$ J: Inferior colliculus in the rat: neuronal responses to stimulation of the auditory cortex. Neurosci Lett 51: 235-240, 1984. 
TAN AY, WEHR M: Balanced tone-evoked synaptic excitation and inhibition in mouse auditory cortex. Neuroscience 163: 1302-1315, 2009.

TAN AY, ATENCIO CA, POLLEY DB, MERZENICH MM, SCHREINER CE: Unbalanced synaptic inhibition can create intensity-tuned auditory cortex neurons. Neuroscience 146: 449-462, 2007.

WINER JA: Decoding the auditory corticofugal systems. Hear Res 212: 1-8, 2006.

WU CH, JEN PH: Neurons in the inferior colliculus of the big brown bat show maximal amplitude sensitivity at the best duration. Chin J Physiol 50: 258-268, 2007.

WU CH, JEN PH: Echo amplitude selectivity of the bat is better for expected than for unexpected echo duration. Neuroreport 20: 1183-1187, 2009.

WU GK, LI P, TAO HW, ZHANG LI: Nonmonotonic synaptic excitation and imbalanced inhibition underlying cortical intensity tuning. Neuron 52: 705-715, 2006.

ZHOU X, JEN PH: Corticofugal inhibition compresses all types of rate-intensity functions of inferior collicular neurons in the big brown bat. Brain Res 881: 62-68, 2000.

ZHOU X, JEN PH: The effect of sound duration on rate-amplitude functions of inferior collicular neurons in the big brown bat, Eptesicus fuscus. Hear Res 166: 124-135, 2002.

ZHOU M, TAO HW, ZHANG LI: Generation of intensity selectivity by differential synaptic tuning: fast-saturating excitation but slow-saturating inhibition. J Neurosci 32: 18068-18078, 2012. 